\title{
Procedimentos de ensino utilizados no curso de Odontologia de uma universidade pública
}

\author{
Natasha de Almeida Dutra Toledo*; Raquel Baroni de Carvalho** \\ * Mestre em Clínica Odontológica, Universidade \\ Federal do Espírito Santo \\ ** Professora Associada, Departamento de Medicina \\ Social, Universidade Federal do Espírito Santo
}

Recebido em 20/03/2018. Aprovado em 12/02/2019.

\begin{abstract}
RESUMO
O processo de ensino-aprendizagem vem passando por muitas mudanças ao longo dos anos, um dos motivos é buscar adaptar-se à geração de estudantes das escolas e universidades. Essa adaptação vem ocorrendo também no currículo dos cursos de nível superior, dentre eles, a Odontologia. Uma forma de registrar tais mudanças é a análise do projeto pedagógico dos cursos (PPC) de Odontologia. No PPC existem informações vitais e importantes sobre o objetivo, o curso, disciplinas obrigatórias e optativas, bem como os procedimentos de ensino que os docentes estão utilizando. O objetivo do presente estudo foi verificar os procedimentos de ensino registrados no PPC do curso de Odontologia da Universidade Federal do Espírito Santo (UFES) e comparar com os procedimentos de ensino citados pelos docentes que atuam no ciclo profissionalizante, por meio de entrevista baseada em roteiro. Na pesquisa documental do PPC foram incluídas $50 \%$ (15) das disciplinas do ciclo profissionalizante $\left(5^{\circ}\right.$ ao $10^{\circ}$ período). A seguir, foram entrevistados os docentes destas disciplinas lotados em três departamentos - Clínica Odontológica, Prótese Dentária e Medicina Social - os quais foram selecionados aleatoriamente. $\mathrm{O}$ procedimento de ensino mais frequentemente citado pelos docentes foi a aula expositiva (93\%), seguido de seminário (40\%). No PPC o procedimento de ensino registrado mais frequentemente foi o seminário (91\%), seguido da aula expositiva (83\%). Outros seis procedimentos de ensino foram citados pelos docentes e registrados no PPC, dentre eles a pesquisa bibliográfica, aprendizado baseado em problema, estudo dirigido, grupo de estudo, discussão e problematização. Existe pouca diversidade nos procedimentos de ensino citados pelos docentes entrevistados e descritos no PPC de Odontologia da UFES. Pode-se concluir que existe alguma dificuldade por parte dos docentes entrevistados para adotar novos procedimentos de ensino.

Descritores: Metodologia. Educação Superior. Docentes. Educação em Odontologia
\end{abstract}

\section{INTRODUÇÃO}

Muitas mudanças ocorreram na sociedade ao longo do tempo, assim como em relação ao processo de ensino-aprendizagem. Para que o ensino evolua a ponto de conseguir envolver os estudantes, novas formas e procedimentos de 
ensino que se mostram eficazes devem ser aplicados. Para verificar sua utilização, a análise dos Projetos Pedagógicos de Curso é fundamental. Por isso, os procedimentos de ensino encontrados no Projeto Pedagógico do Curso de Odontologia (PPC) da Universidade Federal do Espírito Santo (UFES) são o objeto de estudo deste trabalho.

No Brasil, a Lei $n^{\circ}$ 9.394, de 20 dezembro de 1996, estabelece as Diretrizes e Bases da Educação nacional. Um dos princípios e fins da educação é gerar "pluralismo de ideias e de concepções pedagógicas". Com relação ao ensino no nível superior, o Capítulo IV desse documento descreve a educação superior como tendo várias finalidades, dentre elas, a primeira é "estimular a criação cultural e o desenvolvimento do espírito científico e do pensamento reflexivo"1.

Porém, o que se observa hoje é a predominância do currículo tradicional, no qual se espera que o educador passe "uma receita" e os estudantes realizem uma repetição exata na prática. Para que se alcance o ideal, a principal preocupação não deve ser somente com os objetivos cognitivos, a interdisciplinaridade deve ser implantada e junto com ela mudanças no currículo. Como exemplo, o estudante deve ser colocado em contato com a realidade da sua profissão desde o seu primeiro ano universitário, isso significa que o paradigma de que ele só pode se dirigir à prática com pré-requisitos teóricos deve ser quebrado, pois muitas vezes a prática pode anteceder a teoria. Também se tem que possuir ciência de que o conhecimento não é adquirido de forma linear, mas é construído em rede, por isso não é necessário seguir sempre uma sequência ou receita. A aquisição da informação e do conhecimento se dará à medida em que a necessidade de entender determinado tema aparecer $^{2}$.

Nos dias atuais, os procedimentos de ensino que levem em conta as imensas novas possibilidades de aprendizagem são essenciais. Mas, para que sejam empregados, é necessário que os docentes conheçam as novas tendências pedagógicas (incluindo as tecnológicas), saibam como utilizá-las, até mesmo identificando as suas vantagens e limitações. Isso é possível por meio de cursos, autoformação e até mesmo pelo aprendizado que ocorre no dia a dia, por meio de erros e acertos ${ }^{3}$.

A Geração Y ou Geração da Internet, encontrada hoje nas universidades, tem grande expectativa quanto à tecnologia, por isso não é de se admirar que essa expectativa também exista em relação ao ensino. Em um estudo feito com 25 estudantes em uma universidade nos Estados Unidos, foi perguntado qual era a preferência em relação ao nível de interatividade no desenvolvimento do aprendizado. Havia quatro opções: a primeira somente aula convencional; a segunda, $75 \%$ convencional e $25 \%$ interativa; a terceira, $50 \%$ cada; e a quarta, somente interativa. Todos escolheram a terceira opção, onde o convencional e o interativo se misturavam ${ }^{4}$. Essa pesquisa mostrou que os universitários sentem a necessidade de interatividade e tecnologia no seu ambiente de aprendizado, o que mostra a importância de procedimentos de ensino atuais para atrair os discentes que se encontram nas universidades.

Além da Geração Y é importante também mencionar a Geração Z. Esses são os que nasceram entre 1995 e $2010^{5}$. A geração $Z$ tem interesse em selecionar somente aquilo que deseja, se a informação fosse "comparada a um quebracabeça", eles separariam apenas as peças desejadas para sua informação, sem necessidade de montar o quebra-cabeça. Por essa razão, textos informativos longos têm perdido interesse aos olhos desses jovens. Até que se consiga extrair a informação necessária, muito tempo é utilizado. Essa geração foi influenciada, nasceu, cresceu e se desenvolveu em um mundo complexo, veloz e tecnológico ${ }^{6}$.

Em uma pesquisa realizada no curso de Odontologia da Universidade Federal do Espírito 
Santo, constatou-se que $91,1 \%$ dos professores mostraram estar interessados em realizar cursos para desenvolvimento profissional e aperfeiçoar os procedimentos de ensino, pois notavam necessidade de se modificar e atualizar, a fim de atingir a nova geração inserida hoje na universidade ${ }^{7}$.

Assim, o objetivo desse estudo foi verificar quais procedimentos de ensino estão registrados no PPC Odontologia/2013 da UFES e quais são citados pelos docentes do mesmo curso, em entrevista.

\section{METODOLOGIA}

Esta pesquisa faz parte de um projeto aprovado pelo Comitê de Ética em Pesquisa com Seres Humanos do Centro de Ciências da Saúde da UFES sob registro CAAE 339550144.0000.5060, intitulado "Análise do Processo de ensinoaprendizagem na área da saúde".

Foi realizado um estudo documental, exploratório, descritivo e de natureza quantitativa, seguido de entrevistas com os docentes do curso de Odontologia da UFES, realizadas por uma estudante de pós-graduação devidamente preparada para essa função. A pesquisa documental foi realizada analisando os procedimentos de ensino de todas as disciplinas do ciclo profissionalizante do curso descritos no PPC versão 2013, no período de setembro a dezembro de 2015, e os procedimentos de ensino citados foram classificados em individuais ou interativos de acordo com a literatura ${ }^{8}$. Foram excluídas todas as disciplinas do ciclo básico ( $1^{\circ}$ ao $4^{\circ}$ período), pois o objetivo do estudo era avaliar somente o ciclo profissionalizante.

Um roteiro de entrevista foi elaborado a partir dos itens observados no PPC. Realizou-se um teste piloto com professores do ciclo básico do curso com o objetivo de otimizar a utilização do questionário, inserindo questões relevantes e retirando questões irrelevantes.
A seguir, foram entrevistados os docentes que atuavam no ciclo profissionalizante $\left(5^{\circ}\right.$ ao $10^{\circ}$ período), selecionados aleatoriamente entre os departamentos de Clínica Odontológica, Prótese Dentária e Medicina Social, no período de junho de 2016 a setembro de 2017. Destes, 11 concordaram em participar da pesquisa. Os docentes que ministravam mais de uma disciplina, responderam mais de um questionário, um relativo à cada disciplina ministrada. Antes da entrevista, foi apresentado ao docente o Termo de Consentimento Livre e Esclarecido. A primeira etapa da entrevista consistia em três perguntas relacionadas à disciplina, período e procedimentos de ensino utilizados. Foi perguntando, também, se recordavam quais procedimentos de ensino estavam registrados no PPC 2013, e se poderiam citá-los. Após essa etapa, foi feita a leitura dos procedimentos de ensino registrados no PPC para o docente, descritos no tópico Metodologia da respectiva disciplina. Após a leitura, perguntava-se aos docentes se gostariam de acrescentar ou remover algo, além de alguma observação. Não foi realizada a gravação da entrevista, e sim a transcrição de todas as falas dos docentes no questionário.

Após a entrevista, as 15 disciplinas citadas pelos docentes $(50 \%$ das disciplinas do ciclo profissionalizante do curso) foram avaliadas no PPC. Foi realizada uma análise descritiva dos dados por similaridades e porcentagem. Os procedimentos de ensino citados pelos docentes também foram divididos de acordo com a classificação encontrada na literatura $^{8}$ em procedimentos individuais e interativos, sendo os individuais a aula expositiva, o estudo dirigido, a leitura dirigida e a pesquisa bibliográfica. Seminários, discussão e dramatização foram classificados como procedimentos de ensino interativos. Os métodos de problematização, aprendizagem baseada em problema (ABP Problem Based Learning/PBL), sala de aula 
invertida (Flipped Classroom) e gamificação foram considerados como procedimentos de ensino interativos, apesar de não terem sido citados.
Com o intuito de esclarecer e descrever a terminologia utilizada, foi elaborado um glossário de termos relacionados ao tema educação (quadro 1).

\section{Quadro 1. Glossário de termos relacionados ao tema educação}

Aprendizagem. Aquisição de conhecimento ou desenvolvimento de habilidades e atitudes em decorrência de experiências

Aprendizagem Baseada em Problema/ABP (Problem Based Learning/PBL). Procedimento de ensino em que os estudantes trabalham com o objetivo de solucionar um problema. Centrada no estudante, que deixa o papel de receptor passivo e assume o de agente e principal responsável pelo seu aprendizado ${ }^{10}$.

Aula Expositiva. É a apresentação oral de um determinado tema ${ }^{11}$.

Conteúdo Didático. Corresponde aos temas e aos assuntos que serão estudados na disciplina com vistas ao alcance dos objetivos pretendidos ${ }^{10}$.

Didática. Compreende uma parte da pedagogia e preocupa-se com os fundamentos, as condições e as formas de aplicação da instrução e do ensino. A didática estuda a técnica de ensino em todos os aspectos práticos e operacionais. Em uma definição mais simples e romântica, podemos afirmar que a didática é a arte e a ciência de ensinar ${ }^{12}$.

Educação Bancária. Mera transmissão de conhecimentos do professor (educador) para o aluno (educando). Serve como prática para a dominação do ensino por parte do professor, nega a dialogicidade, funciona como depósito e transferência de valores e conhecimentos do professor para o aluno, não gera criatividade, transformação e saber ${ }^{13}$.

Educação. Uma tentativa constante de mudança de atitude, que desenvolva no estudante o gosto pela pesquisa, um ato de amor e coragem, e uma afirmação da liberdade ${ }^{14}$.

Ensino. É uma forma sistemática de transmissão de conhecimentos utilizada pelos humanos para instruir e educar seus semelhantes, geralmente em locais conhecidos como escolas ${ }^{15}$. De acordo com Paulo Freire ${ }^{16}$, ensinar não é transferir conhecimento, mas criar as possibilidades para a sua produção ou a sua construção.

Estratégias de ensino-aprendizagem/Métodos/Técnicas/Meios/Procedimentos de Ensino. Para facilitar a aprendizagem, o professor se vale de estratégias, ou seja, da aplicação dos meios disponíveis com vistas à consecução dos seus objetivos ${ }^{9}$.

Estudo Dirigido. Visa primordialmente ensinar a estudar e a aprender ${ }^{11}$.

Ferramentas de Ensino. Tecnologias inseridas nas formas de aplicar conteúdo ${ }^{17}$.

Gamificação. Utilizar mecanismos de jogos para tornar o aprendizado mais divertido e interativo ${ }^{18}$.

Geração Y. Indivíduos que nasceram entre 1980 e 2000, também pode ser chamada de Geração da Internet ou Milênios. Geralmente possuem facilidade com a tecnologia e dificuldade com modelos tradicionais de ensino, buscando métodos não convencionais ${ }^{19}$.

Geração Z. Comumente abreviada para Gen Z, também conhecida como iGeneration, Plurais ou Centennials. É a definição sociológica para definir a geração de pessoas nascidas de 2000 até 2010. A teoria mais aceita por estudiosos é que essa geração surgiu como concepção para suceder a Geração Y. Portanto, é a geração que corresponde à idealização e nascimento da internet, criada em 1990 e no boom da criação de aparelhos tecnológicos (nascidos entre o começo de 2000 a 2010). A grande nuance dessa geração é “zapear" entre canais de televisão, internet, vídeogame e smartphones 5 .

Leitura Dirigida. Apresentação de temas e ideias por meio de textos didáticos na sala de aula ${ }^{11}$.

Pesquisa Bibliográfica. Dá ênfase à literatura para obtenção dos dados necessários à explicação e compreensão do assunto ${ }^{12}$.

Problematização. Processo de busca por uma temática significativa, com problemas a serem decifrados, jamais como conteúdos a serem depositados ${ }^{11}$.

Processo Ensino-Aprendizagem. Os alunos como os principais agentes do processo educativo. Envolve identificar suas aptidões, necessidades e interesses com vistas a auxiliá-los na coleta de informações de que necessitam no desenvolvimento de novas habilidades, na modificação de atitudes e comportamentos e na busca de novos significados para as pessoas, coisas e fatos $^{10}$.

Projeto Pedagógico. É o instrumento de concepção de ensino e aprendizagem de um curso e apresenta características de um projeto, no qual devem ser definidos os seguintes componentes: 1. Concepção do Curso. 2. Estrutura do Curso: currículo, corpo docente, corpo técnicoadministrativo e infraestrutura. 3. Procedimentos de avaliação dos processos de ensino e aprendizagem e do curso. 4. Instrumentos normativos de apoio (composição do colegiado, procedimentos de estágio, TCC, entre outros.) ${ }^{20}$.

Recursos de ensino. Existe uma grande variedade de recursos de ensino, desde os mais simples, como quadro de giz, cartazes, álbuns seriados, reprodução de textos, até os mais complexos, como câmera de vídeo e projetor multimídia ${ }^{10}$.

Sala de aula invertida (Flipped Classroom). Fornecer informações e materiais para o aluno previamente, para que ele possa discutir o assunto com os colegas e com o professor na sala de aula ${ }^{21}$.

Seminário. Um grupo de pessoas que se reúne sob a coordenação de alguém que é especialista, para discutir determinado tema? 


\section{RESULTADOS}

Dentre os participantes, $86 \%$ responderam que tinham conhecimento das metodologias/ procedimentos de ensino registrados no PPC das suas disciplinas. Porém, os resultados desta pesquisa indicam que os procedimentos de ensino relatados pelos docentes na entrevista não estão de acordo com aqueles que acreditavam estar descritos no PPC, nem com os procedimentos de ensino efetivamente registrados no documento. Como pode ser observado na tabela 1, apesar de algum dos procedimentos de ensino citados pelos docentes coincidirem com os registrados no PPC, em nenhuma das entrevistas a totalidade dos procedimentos de ensino registrados no PPC foi mencionada pelos docentes.

Os resultados abrangem $50 \%$ das disciplinas do ciclo profissionalizante do curso de Odontologia da UFES, totalizando 15 disciplinas, conforme pode ser observado no quadro 2. Nesse quadro também pode-se encontrar a classificação dos procedimentos de ensino de acordo a literatura ${ }^{8}$, citados pelos docentes na entrevista.

Tabela 1. Procedimentos de ensino registrados no PPC, citados pelos docentes na entrevista e coincidência entre eles

\begin{tabular}{lccc}
\hline Procedimentos de ensino & $\begin{array}{c}\text { Citados } \\
\text { no PPC } \\
\mathbf{n}(\boldsymbol{\%})\end{array}$ & $\begin{array}{c}\text { Citados por } \\
\text { docentes } \\
\mathbf{n}(\boldsymbol{\%})\end{array}$ & $\begin{array}{c}\text { Disciplinas com coincidência de procedimentos } \\
\text { citados no PPC e pelos docentes } \\
\text { n }(\boldsymbol{\%})\end{array}$ \\
\hline Aula expositiva & $10(83 \%)$ & $14(93 \%)$ & $9(60 \%)$ \\
Seminário & $11(91 \%)$ & $6(40 \%)$ & $5(33 \%)$ \\
PBL ou APB & $1(8 \%)$ & $1(6 \%)$ & - \\
Estudo Dirigido & $1(8 \%)$ & $3(20 \%)$ & - \\
Grupo de Estudo & $1(8 \%)$ & - & - \\
Técnica de Pesquisa & $6(40 \%)$ & - & - \\
Discussão & - & $2(13 \%)$ & - \\
Resolução de Problemas (Problematização) & - & $1(6 \%)$ & - \\
\hline
\end{tabular}

Quadro 2. Classificação dos procedimentos de ensino em Individuais ou Interativos

\begin{tabular}{|l|l|}
\hline Procedimentos de ensino Individuais registrados no PPC & Procedimentos de ensino Individuais citados na entrevista \\
\hline $\begin{array}{l}\text { Aula expositiva } \\
\text { Estudo Dirigido } \\
\text { Técnica de Pesquisa }\end{array}$ & $\begin{array}{l}\text { Aula expositiva } \\
\text { Estudo Dirigido }\end{array}$ \\
\hline Procedimentos de ensino Interativos registrados no PPC & Procedimentos de ensino Interativos citados na entrevista \\
\hline Grupo de Estudo & Seminário \\
Seminário & PBL ou APB \\
PBL ou APB & Discussão \\
& Resolução de Problemas (Problematização) \\
\hline
\end{tabular}

O procedimento de ensino mais citado pelos docentes foi a aula expositiva, seguido do seminário. No PPC, observou-se que o procedimento de ensino mais citado é o seminário, seguido da aula expositiva. A ABP foi citada apenas uma vez, tanto no PPC quanto na entrevista. $\mathrm{O}$ estudo dirigido foi citado na entrevista por três docentes, enquanto que só foi registrado uma vez no PPC. O grupo de estudo foi registrado apenas uma vez no PPC, enquanto que a discussão e a problematização foram citadas apenas na entrevista. A discussão foi citada por dois docentes e a problematização, por um docente. Confrontando as informações fornecidas pelos 
docentes na entrevista e as informações registradas no PPC, observam-se apenas oito procedimentos de ensino diferentes.

$\mathrm{Na}$ entrevista, muitos docentes mencionaram como procedimentos de ensino o que a literatura ${ }^{9,11}$ classifica como recursos e ferramentas de ensino, a saber: artigos, anotações no caderno, computação, exercícios, internet, lousa, manequins, portal do aluno, relatório, slides, vídeo e Whats App. Além disso, o conteúdo didático também foi citado por alguns docentes na entrevista como procedimento de ensino: instrumentação cirúrgica, instrumentação manual sônica e ultrassônica, técnicas de afiação e raspagem, periodontograma, instrumentação manual e rotatória, terapêuticas não cirúrgicas, instrumentações cirúrgicas e não cirúrgicas periodontais.

Das 15 disciplinas analisadas, uma não estava presente no PPC e outras duas não apresentavam os procedimentos de ensino relatados: uma não apresentava o tópico Metodologia, e a outra, apesar de apresentar o tópico, não descrevia os procedimentos de ensino utilizados.

\section{DISCUSSÃO}

$\mathrm{O}$ fato de ter-se encontrado no presente estudo a aula expositiva como o procedimento de ensino mais citado pelos docentes e o segundo mais registrado no PPC não é surpresa ${ }^{4,9,11}$. Na Faculdade de Odontologia da Universidade Federal de Minas Gerais as aulas expositivas foram encontradas em $96,7 \%$ dos planos de ensino ${ }^{8}$.

Uma pesquisa qualitativa realizada com professores e alunos do curso de Odontologia da Universidade Estadual de Londrina traz alguns temas vinculados ao processo de ensinoaprendizagem. Seus relatos indicaram uma grande ênfase no processo de ensino centrado no professor como principal agente e responsável pelo ensino e uma quase omissão ao processo de aprendizagem, mostrando uma total predominância do modelo de ensino tradicional. Nos relatos dos docentes, a mesma abordagem tradicional centrada no professor foi encontrada; predominando a autoridade do professor que exige a receptividade dos estudantes e impedindo qualquer comunicação com eles durante a aula. Os professores, assim, ficam responsáveis por aplicar as metodologias, avaliações e conteúdo sem nenhum conhecimento prévio dos alunos ${ }^{22}$.

Ainda corroborando esses dados, um estudo de natureza qualitativa utilizando a técnica de grupo focal para coleta de dados com estudantes do curso de Odontologia da Universidade Federal do Rio Grande do Norte foi realizado em 2012, visando avaliar a percepção dos estudantes em relação ao processo de ensino-aprendizagem em sua universidade. Alguns temas surgiram e foram categorizados, dentre eles podemos citar a metodologia adotada pelo professor. Em relação a esse tema observa-se nos relatos a presença da estratégia de ensino tradicional onde o aluno só recebe aquilo que o professor oferece ${ }^{23}$.

O seminário foi o procedimento de ensino mais registrado no PPC e o segundo mais citado pelos docentes na entrevista, o que é positivo, pois se trata de procedimento de ensino interativo, que estimula a participação dos envolvidos. Porém, cabe ressaltar que, para que um seminário seja efetivo, deve ser mais uma fonte de ideias do que propriamente um meio de informação. Mais importante do que expor o tema é se criar condições para a sua discussão. O professor primeiramente assume o papel de coordenador de discussões e no final expõe seus comentários de natureza crítica e orientadora $^{9}$. Ou seja, uma simples exposição de um tema por meio de projeções de slides não pode ser considerada um seminário, pois não gera debate e discussão. Assim, uma avaliação de como os seminários estão sendo realizados é de primordial importância.

A pesquisa bibliográfica foi mencionada no PPC em 40\% das disciplinas, mas os docentes não 
a citaram nenhuma vez. A pesquisa deve sempre ter a origem em uma situação de necessidade imediata ou prevista. Ao se constatar a necessidade, iniciase então o processo de reflexão sobre o tema. $\mathrm{O}$ professor deve estar atento a ensinar as quatro partes principais da pesquisa, a introdução, o desenvolvimento do trabalho, as conclusões e a bibliografia $^{12}$.

A ABP foi citada apenas uma vez. Sabe-se que esta metodologia visa a um processo de autoaprendizagem, no qual parte de um problema apresentado pelo professor deverá ser resolvido. Assim, o estudante mostra quais são os objetivos a serem aprendidos, esclarecendo os termos, analisando o problema, dando prováveis hipóteses de solução, criando os objetivos da aprendizagem, procurando fontes de conhecimento para que possa adquirir informações pertinentes, administrando essas informações e conhecimentos para verificar se as suas hipóteses e soluções estavam corretas ${ }^{2}$. Esse é um procedimento de ensino que combina com a geração Y que se encontra atualmente nas universidades.

Entretanto, foram observados resultados divergentes em relação à aplicação da $\mathrm{ABP}$ em um estudo observacional realizado com alunos da disciplina de Radiologia Odontológica de uma instituição de ensino privada na Região Norte do Brasil. Participaram do estudo 138 estudantes; $92 \%$ deles consideravam válida a experiência com novos procedimentos de ensino e $93,53 \%$ não conheciam o método de ABP. Quando questionados sobre o método de sua preferência para aprendizado, venceu o método tradicional, pois consideraram que teriam um melhor aproveitamento da disciplina e não gostariam de estudar em uma universidade na qual todas as disciplinas utilizassem ABP. Considerou-se que esse dado poderia advir do fato de os estudantes não estarem familiarizados com a técnica e que um grande número ficar insatisfeito com a pontuação final obtida na avaliação ${ }^{24}$.
A problematização foi citada apenas uma vez por um docente, que mencionou utilizar a metodologia de "resolução de problemas". Por essa definição, não se pode dizer ao certo se o docente estava se referindo à $\mathrm{ABP}$ ou à problematização, pois as duas envolvem o aprendizado com problemas. Porém, como o problema é dado pelo professor, entendemos que o método citado poderia ser classificado como problematização. É interessante reforçar que esse procedimento de ensino não foi mencionado por nenhuma disciplina no PPC, apesar de esse mesmo documento citar que um dos objetivos do curso de Odontologia é desenvolver novas técnicas de ensino baseadas na problematização.

O estudo dirigido foi citado apenas uma vez tanto na entrevista como no PPC, e possui, como todo procedimento de ensino, as suas vantagens e desvantagens. Pode-se citar como vantagens incentivar hábitos de estudos, favorecer sentimento de independência e segurança. Contudo, quando os exercícios são mecânicos, não se enquadram nesta metodologia ${ }^{25}$.

A discussão, apesar de ter sido citada por docentes de apenas duas disciplinas e de não estar presente no PPC, tem um grande valor pedagógico. Ela representa um exercício de liberdade e tem sido recomendada como um dos procedimentos de ensino mais importantes como alternativa à aula expositiva. Porém, se não for bem conduzida, alguns efeitos indesejados podem ser vistos, como o baixo nível de participação dos alunos, fuga dos objetivos e animosidade entre os alunos ${ }^{9}$.

$\mathrm{O}$ grupo de estudo, citado por apenas um docente, vem se revelando um excelente meio de instruir e formar socialmente o estudante. Promove a sociabilidade, várias opiniões sobre um mesmo tema, surgimento de líderes, espírito comunitário e o desenvolvimento de habilidades para trabalhar com outras pessoas. Esse tipo de técnica de ensino requer um bom planejamento para ser executado de forma completa e eficaz ${ }^{12}$. 
Apenas oito diferentes procedimentos de ensino foram mencionados, levando em consideração a entrevista e a análise documental, um número muito pequeno. No estudo realizado na $\mathrm{UFMG}^{8}$ foram encontrados 11 diferentes procedimentos somente no projeto pedagógico e os autores concluíram que existe pouca diversidade nos procedimentos de ensino das disciplinas analisadas.

Cabe ressaltar que a diversidade dos procedimentos de ensino registrados no PPC do curso de Odontologia da UFES poderia ter sido maior se estivessem registrados em todas as disciplinas analisadas, o que aponta para a necessidade da elaboração de um novo PPC que corrija erros existentes e que apresente novos procedimentos de ensino interativos e inovadores.

Ainda, pode-se levar em consideração que muitos docentes citaram conteúdos didáticos, recursos e ferramentas de ensino como procedimentos de ensino. Esse resultado mostra a necessidade de melhor qualificação dos docentes na área pedagógica. A literatura ${ }^{7}$ alerta para essa necessidade, ao apontar que os cursos de pós-graduação devem mostrar aos futuros professores diferentes abordagens e métodos de ensino existentes. Além disso, os próprios docentes precisam despertar para a necessidade de incentivar o discente a aprender a refletir e construir de forma crítica o conhecimento.

Em relação aos procedimentos de ensino individuais e interativos, em seis disciplinas diferentes os docentes só mencionaram utilizar procedimentos individuais, ou seja, $40 \%$ das disciplinas. Porém, os dados analisados no PPC foram que somente uma disciplina apresenta unicamente procedimentos de ensino tradicionais. Das disciplinas analisadas no estudo na $\mathrm{UFMG}^{7}$ no PPC do curso de Odontologia foi registrado que $20 \%$ também só apresentaram procedimentos de ensino individuais.

\section{CONCLUSÃO}

Nenhum docente entrevistado demonstrou conhecer totalmente os procedimentos de ensino de sua disciplina registrados no PPC 2013. Foi verificada certa dificuldade por parte dos docentes entrevistados quanto ao entendimento das diferenças entre as definições, terminologias de procedimentos de ensino, conteúdo didático, recursos e ferramentas de ensino.

\section{ABSTRACT \\ Teaching procedures used in the Dentistry program of a public university}

The teaching-learning process has undergone many changes over the years, one of the reasons is to seek to adapt to the generation of students from schools and universities. This adaptation has occurred also in the curriculum of higher education programs, including Dentistry. A way to register such changes is by analyzing the Dentistry Program Pedagogic Project (PPP). The PPP contains vital and important information about the objective, the program, compulsory and optional courses, as well as the teaching procedures that professors are using. The objective of this study was to analyze the teaching procedures registered in the Federal University of Espírito Santo (UFES) Dentistry Program PPP and compare them with the teaching procedures cited by professors working in the professional training cycle through questionnaire-based interview. In the documentary research of the PPP we included $50 \%$ (15) of the courses of the professional training cycle (5th to 10 th period). Then, we interviewed the professors of these courses working in three departments - Clinical Dentistry, Prosthodontics and Social Medicine which were randomly selected. The teaching procedure most often cited by professors was lecture $(93 \%)$, followed by seminar $(40 \%)$. The teaching procedure registered most often in the PPP was seminar $(91 \%)$, followed by lecture (83\%). Six other teaching procedures were cited by professors and registered in the PPP, among them bibliographical research, problem based learning, directed study, group study, discussion, 
and problematization. There is little diversity in the teaching procedures cited by the interviewed professors and described in the UFES Dentistry PPP. It can be concluded that there is some difficulty on the part of the interviewed professors as to adopting new teaching procedures.

Descriptors: Methodology. Higher Education. Faculty. Dental Education.

\section{REFERÊNCIAS}

1. Ministério da Educação e Cultura. Secretaria de Educação Superior, Lei de Diretrizes e Bases da Educação Nacional: Lei n ${ }^{\circ} 9.394$ (1996).

2. Masetto MT. Um paradigma interdisciplinar para a formação do cirurgião-dentista. In: Carvalho ACP, Kriger L. São Paulo: Artes Médicas Ltda; 2006. p. 31-50.

3. Feldkercher N, Mathias CV. Uso das TICs na Educação Superior presencial e a distância: a visão dos professores. Rev Iberoam Tecnol en Educ y Educ en Tecnol. 2011;6:84-91.

4. Oblinger DG, Oblinger JL. Educating the next generation. Science and Justice. Boulder: Educause; 2005. [Acesso em 05 de setembro de 2017]. Disponível em: www.educause.edu/educatingthenetgen/

5. Wikipédia, a enciclopédia Livre. Flórida: Wikimedia Foundation; 2018 [Acesso em 07 de fevereiro de 2018]. Disponível em: https://pt.wikipedia.org/w/index.php?title= Gera\%C3\%A7\%C3\%A3o_Z\&oldid=51186 192

6. Faber, Jorge. A Geração Z e a evolução das revistas científicas. Dental Press J Orthod. 2011;16(4):7.

7. Farias CM. Análise do processo de ensino e de aprendizagem no ciclo profissionalizante do curso de odontologia da UFES: percepção de docentes e estudantes.
[Dissertação]. Vitória: Universidade Federal do Espírito Santo; 2015.

8. Senna MIB, Rückert B, Pinto MJB, Lucas SD. Procedimentos de ensino adotados no curso de graduação em Odontologia da Universidade Federal de Minas Gerais : uma análise documental. Arq Odontol. 2015;51(3):129-37.

9. Gil AC. Metodologia do Ensino Superior. 4th. São Paulo: Atlas; 2011.

10. Gil AC. Didática do Ensino Superior. 1 ed. São Paulo: Atlas, 2011.

11. Marques, Heitor Romero. Metodologia do Ensino Superior. 5 ed. Campo Grande: UCDB, 2015.

12. Consolaro A. O "Ser" Professor: Arte e Ciência no Ensinar e Aprender. 3 ed. Maringá: Dental Press; 2002.

13. Freire, Paulo. Pedagogia do Oprimido. 17 ed. Rio de Janeiro: Paz e Terra LTDA, 1987.

14. Freire, Paulo. Educação como prática da Liberdade. Rio de Janeiro: Paz e Terra LTDA, 1967.

15. Wikipédia. Ensino. Flórida: Wikimedia Foundation; 2018 [Acesso em 07 de fevereiro de 2018]. Disponível em: https://pt.wikipedia.org/w/ index.php?title= Ensino\&oldid $=5093540$.

16. Freire, Paulo. Pedagogia da autonomia: saberes necessários à prática educativa. São Paulo: Paz e Terra, 1996.

17. Elsevier. 5 ferramentas de ensino que irão facilitar sua metodologia. Rio de Janeiro: Elsevier Editora LTDA [Acesso em 07 de fevereiro de 2018]. Disponível em: https://www.elsevier.com.br/elsevier-maisprofessor/blog/5-ferramentas-de-ensino-queirao-facilitar-sua-metodologia/.

18. Pfeiffer. Hoboken, NJ. The Gamification of learning and instruction: Game-based methods and strategies for training and education. 1. ed. Washington: Pfeiffer \& 
Company, 2012.

19. Gazola, André Augusto. A Geração Y: Desafios para educação dos Nativos Digitais. Caxias do Sul: Lendo.org [Acesso em 07 de fevereiro de 2018]. Disponível em: https://www.lendo.org/geracao-y-caracteris ticas-educacao/.

20. Universidade Federal de Minas Gerais. PróReitoria de Graduação: Projeto Pedagógico de Curso. Belo Horizonte: UFMG [Acesso em 07 de fevereiro de 2018]. Disponível em: https://www.ufmg.br/prograd/arquivos/dest aque/ppc.pdf.

21. Pacheco, José. Sala de aula invertida. Rev Educação. 2014:14-17.

22. Lazzarin HC. O papel do professor no processo ensino-aprendizagem: percepção de professores e alunos de Odontologia. [Tese]. Londrina: Universidade Estadual de Londrina; 2005.
23. Noro LRA, Farias-Santos C de S, Sette-deSouza PH, Pinheiro IAG, Borges REA, Nunes LMF, et al. O professor (ainda) no centro do processo ensino-aprendizagem em Odontologia. Rev ABENO. 2015;15(1):211.

24. Galvão S, Azevedo-Vaz SL de, Oliveira ML. $\mathrm{O}$ método de aprendizagem baseada em problemas na disciplina de Radiologia Odontológica. Rev ABENO. 2016;16(4):72-8.

25. Carvalho J. Metodologia do Ensino Superior. Vitória: EDUFES; 1998.

\section{Correspondência para:}

Natasha de Almeida Dutra Toledo

e-mail: natashatoledo@outlook.com.br

Rua Carlos Alves, 200/1402, Bento Ferreira 29050-040 Vitória/ES 